\title{
COMPOSIÇÃO QUÍMICA E ATIVIDADE ANTIOXIDANTE DA POLPA E RESÍDUOS DE ABACATE 'HASS'
}

\author{
ÉRICA REGINA DAIUTO², MARIA AUGUSTA TREMOCOLDI ${ }^{3}$, \\ SEVERINO MATIAS DE ALENCAR ${ }^{4}$, ROGÉRIO LOPES VIEITES ${ }^{5}$, \\ PHILLIP HERBEST MINARELLI ${ }^{6}$
}

RESUMO- $O$ abacate é um fruto muito nutritivo, sendo a polpa a principal parte utilizada para consumo in natura, na forma de sobremesa, saladas, molhos e cosméticos, além de ser utilizada para extração de óleo. Os resíduos, casca e semente, ainda são pouco explorados cientificamente quanto ao seu potencial nutritivo e funcional. Neste trabalho, amostras liofilizadas da casca, polpa e semente de abacate 'Hass' foram submetidas a análises de composição centesimal, minerais, teor de compostos fenólicos totais e capacidade antioxidante. A polpa apresentou elevado teor de lipídios em relação à casca e à semente do abacate. Os elementos minerais foram superiores na casca dos abacates. A casca e a semente possuem maior teor de compostos fenólicos e atividade antioxidante em relação à polpa de abacate.

Termos para indexação: Persea americana Mill, minerais, casca, semente, propriedade funcional.

\section{CHEMICAL COMPOSITION AND ANTIOXIDANT ACTIVITY OF THE PULP, PEEL AND BY PRODUCTS OF AVOCADO 'HASS}

\begin{abstract}
The avocado is a very nutritious fruit being the pulp the main part used for fresh consumption, in the dessert form, salads, sauces and cosmetics, besides being used for oil extraction. The residues, peel and seed are still little explored scientifically as this nutritious and functional potential. In this work, freeze dryied samples of the pulp, peel and seed of the 'Hass' avocado were submitted to analyses of centesimal composition, minerals, total phenolic content and antioxidant capacity. The pulp presented high content of lipids in relation to avocado peel and seed. The mineral elements were superior in the avocados peel. The peel and the seed possess larger total phenolic content and antioxidant activity in relation to the pulp.

Index terms: Persea americana Mill., minerals, pell, seed, functional property
\end{abstract}

\section{INTRODUÇÃO}

$\mathrm{O}$ abacate (Persea americana Mill.) possui notável qualidade nutricional, pois contém grande quantidade de vitaminas, minerais, proteínas e fibras, além do elevado teor de lipídios que confere destaque ao fruto como auxiliar na prevenção de doenças cardiovasculares. Além disso, contém níveis elevados de compostos fitoquímicos bioativos, incluindo a vitamina $\mathrm{E}$, carotenoides, esteróis, compostos fenólicos, entre outros (LEE et al., 2004).

O abacate 'Hass', denominado 'avocado', destaca-se dentre as variedades comercializadas no mercado brasileiro, sendo de menor diâmetro, casca mais grossa, maior teor de lipídios, menor teor de água e, portanto, apresentando uma polpa mais consistente. Porém ainda a maior parte da produção do 'avocado' é destinada ao mercado externo.

O valor comercial e nutricional do 'avocado' tem sido demonstrado por vários estudos na área de pós- colheita e processamento de abacate, tendo por objetivo a comercialização do fruto com suas qualidades preservadas, agregação de valor e novas formas de consumo. Os produtos de maior interesse comercial derivam da polpa de abacate, tais como óleo, polpas, guacamole e pastas.

A proporção de casca, polpa e semente presentes no Abacate 'Hass' é de, respectivamente,

\footnotetext{
${ }^{1}$ (Trabalho 102-13). Recebido em: 17-01-2014. Aceito para publicação em: 01-03-2013. Projeto Financiado pela CAPES e FAPESP. 2Pós- doutoranda da Faculdade de Ciências Agronômicas da UNESP-Botucatu-SP. E-mail: erdaiuto@uol.com.br

${ }^{3}$ Doutoranda Centro de Energia Nuclear na Agricultura, Universidade de São Paulo (USP), 13416-000, Piracicaba-SP, E-mail:mariaaugustatremocoldi@yahoo.com.br

${ }^{4}$ Prof. Dr. Departamento de Agroindústria, Alimentos e Nutrição da Escola Superior de Agricultura Luiz de Queiroz (ESALQ/USP) E-mail: alencar@esalq.usp.br

${ }^{5}$ Docente Departamentos de Horticultura da Faculdade de Ciências Agronômicas da UNESP-Botucatu-SP. E-mail: vieites@fca.unesp.br ${ }^{6}$ Aluno de no curso de Graduação em Engenharia Agronômica da Faculdade de Ciências Agronômicas (FCA/UNESP), Botucatu-SP, E-mail: hminareli@hotmail.com
} 
28,13; 58,71 e 13,16\%, segundo Daiuto et al. (2012), porém Salgado et al. (2008) encontraram valores de $11,2 \% ; 66,0 \%$ e $22,8 \%$, respectivamente, na variedade Margarida. Segundo os mesmos autores, no Brasil, a variedade em estudo tem sido comercializada com selo de certificação para os países europeus e existe interesse por parte de algumas empresas na elaboração de produtos utilizando excedentes e refugos de produção. Tango et al. (2004) apontam que as sementes constituem uma grande porção dos frutos e que, dessa forma, torna-se interessante caracterizálos, visando a futuros estudos sobre o aproveitamento desse volumoso subproduto. Após caracterização da casca e semente, uma das formas de aproveitamento destes resíduos poderia ser feita por meio da extração de compostos biologicamente ativos para uso nas indústrias alimentícias e farmacêuticas.

Embora o abacate seja uma fruta nutritiva, pouco ainda se sabe sobre sua composição fitoquímica e propriedades antioxidantes. Merece destaque o estudo feito por Wang et al. (2010), onde foram avaliadas a capacidade antioxidante e o teor de compostos fenólicos totais de sete cultivares de abacate (Slimcado, Booth 7, Booth 8, Choquette, Loretta, Simmonds e Tonagge) em relação ao abacate 'Hass'. Os autores verificaram que em todas as cultivares, as sementes apresentaram a maior capacidade antioxidante e teor de compostos fenólicos totais em relação à polpa.

A crescente demanda social por produtos que contribuam com a melhoria da qualidade de vida, provenientes especialmente de fontes naturais, aliada à preocupação do setor industial na tentativa de atender a essa exigência, tem impulsionado pesquisas na busca de novas tecnologias, visando à promoção da saúde dos consumidores e, ao mesmo tempo, à diminuição de perdas econômicas e do impacto da atividade industrial ao meio ambiente (MELO, 2010). Neste sentido, pesquisas por novas substâncias naturais bioativas, dentre as quais antioxidantes naturais, têm aumentado significativamente nos últimos anos e os benefícios à saúde cada vez mais elucidados e disseminados (WENG; WANG, 2000; JAYAPRAKASSHA; PATIL, 2007).

Além disto, informações nutricionais de partes não convencionais de alimentos são escassas e podem possuir um papel importante em dietas balanceadas, auxiliando na suplementação da alimentação de populações subnutridas como uma fonte de cálcio, ferro, vitamina $\mathrm{C}$, proteína, fibra, carboidrato e outros componentes nutricionais (PINTO et al., 2000).

Portanto, o objetivo desta pesquisa foi avaliar a composição química, os compostos fenólicos totais e a atividade antioxidante da casca, da polpa e da semente de abacates 'Hass'.

\section{MATERIAL E MÉTODOS}

\section{Matéria- prima}

Foram utilizados frutos de abacate 'Hass' da safra de 2011, fornecidos pela empresa Jaguacy, localizada em Bauru-SP. Os frutos foram cuidadosamente colhidos no ponto de maturação fisiológica (de acordo com o teor de óleo, 21,6\%). A seguir, foram selecionados visando à homogeneização do lote quanto ao tamanho, cor e ausência de injúrias e defeitos, e foram separados manualmente em polpa, casca e semente.

Em seguida, foram congelados, liofilizados no liofilizador L101, da marca Liotop, homogeneizados e armazenados a $-18^{\circ} \mathrm{C}$, até o momento das análises. Foram utilizados 3 lotes de frutos de abacate 'Hass', contendo 20 frutos em cada lote, colhidos ao acaso, sendo utilizados 60 frutos para o experimento.

\section{Análises}

A casca, a polpa e a semente de abacate liofilizada foram caracterizadas por métodos físico-químicos, segundo Normas do Instituto Adolfo Lutz (2008), por meio da determinação dos teores de umidade, proteínas, lipídios, fibra bruta e cinzas. O teor de açúcares totais foi determinado pelo método de Somogy (1945) e Nelson (1944). A acidez titulável, expressa em gramas de acido cítrico.100g polpa ${ }^{-1}$, e o potencial hidrogeniônico foram determinados seguindo as Normas Analíticas do Instituto Adolf Lutz (2008). A determinação da umidade foi realizada também na polpa, casca e semente in natura.

Os elementos $\mathrm{N}, \mathrm{P}, \mathrm{K}, \mathrm{Ca}, \mathrm{Mg}, \mathrm{S}, \mathrm{B}, \mathrm{Fe}, \mathrm{Cu}$, $\mathrm{Mn}$ e $\mathrm{Zn}$ foram determinados por Espectrofotometria de Absorção Atômica, segundo metodologia estabelecida por Malavolta et al. (1997). As análises físico-químicas e dos elementos minerais foram determinadas em triplicata.

Os extratos para análise de compostos fenólicos e a atividade antioxidante foram obtidos em triplicata, como descrito por Kähkönen et al. (1999), com modificações. Inicialmente, pesou-se 1 grama do material liofilizado, moído em moinho análitico A11 Basic, da marca IKA, e adicionaramse $10 \mathrm{~mL}$ dos solventes etanol:água $(80: 20 \mathrm{v} / \mathrm{v})$. A extração foi conduzida em ultrassom, à temperatura ambiente, durante 15 minutos. Após isso, o extrato foi centrifugado a $5.000 \mathrm{xg}$ durante 15 minutos, e o sobrenadante, utilizado para as análises.

A análise do teor de compostos fenólicos totais foi feita de acordo com o método espectrofotométrico 
de Folin-Ciocateau descrito por Singleton et al. (1999), utilizando ácido gálico como padrão. Os extratos foram diluídos e uma alíquota de $0,5 \mathrm{~mL}$ da amostra foi misturada com $2,5 \mathrm{~mL}$ do reagente FolinCiocalteau (diluído 1:10) e com $2 \mathrm{~mL}$ de carbonato de sódio a $4 \%$. Após $2 \mathrm{~h}$ de incubação no escuro à temperatura ambiente, a absorbância foi medida em espectrofotômetro (UV Mini 1240) a $740 \mathrm{~nm}$. Os resultados do teor de compostos fenólicos totais foram expressos em equivalente de ácido gálico, com base em uma curva de calibração com concentrações variando de 5 a $80 \mu \mathrm{g} / \mathrm{mL}$.

A medida da atividade sequestradora do radical DPPH foi realizada de acordo com a metodologia descrita por Mensor et al. (2001). Os extratos foram diluídos, e a mistura de reação foi constituída pela adição de $500 \mu \mathrm{L}$ dos extratos, 3,0 $\mathrm{mL}$ de etanol a $99 \%$ e $300 \mu \mathrm{L}$ do radical DPPH em solução de etanol a $0,5 \mathrm{mM}$ e incubada por 45 minutos, em temperatura ambiente e ao abrigo da luz. A absorbância foi determinada a $517 \mathrm{~nm}$. Os resultados foram expressos em equivalente de trolox, com base em uma curva de calibração de trolox com concentrações variando de 0 a $200 \mu \mathrm{M}$.

Para a determinação da atividade antioxidante, foi empregado o método do radical ABTS [2,2'-azinobis(3-etilbenzotiazolina-6-ácido sulfônico), sendo utilizada a metodologia descrita por Re et al. (1999). Inicialmente, o radical $\mathrm{ABTS}^{++}$ foi formado pela reação de ABTS a $7 \mathrm{mM}$ com persulfato de potássio a $140 \mathrm{mM}$, os quais foram incubados à temperatura ambiente e na ausência de luz, por 16 horas. Transcorrido esse tempo, a solução foi diluída em etanol até a obtenção de uma solução com absorbância de $0,70( \pm 0,01)$. Em ambiente escuro, um volume de $3,0 \mathrm{~mL}$ da solução de radical ABTS • + foi acrescentado a $30 \mu \mathrm{L}$ de cada diluição dos extratos, e as absorbâncias, lidas após seis minutos em espectrofotômetro a $734 \mathrm{~nm}$. Para a construção da curva-padrão, foi utilizado o Trolox, um antioxidante sintético análogo à vitamina $\mathrm{E}$, nas concentrações de 100 a $2000 \mu \mathrm{M}$. Os resultados da atividade antioxidante foram expressos em $\mu \mathrm{M}$ trolox/g.

\section{Metodologia Estatística}

As análises estatísticas dos resultados foram realizadas por meio do software STATISTICAL ANALYSIS SYSTEM (SAS, 2002). Para a comparação das médias, utilizou-se do teste de Tukey, a 5\% de probabilidade.

\section{RESULTADOS E DISCUSSÃO}

\section{Análises físico-químicas}

De acordo com a composição da polpa do abacate, pode-se destacar o teor elevado de lipídios (Tabela 1). De fato, alguns autores apontam o potencial do fruto para a extração de óleo (GOMEZLÓPEZ, 2002; TANGO et al., 2004), inclusive para utilização na indústria alimentícia (SALGADO et al., 2008). Os teores encontrados neste trabalho foram superiores aos relatados por Daiuto et al. (2010), que encontraram valores de lipídios na polpa fresca de $25,2 \%$ e Tango et al. (2004) $31,1 \%$. Porém, isto pode ser devido ao fato da análise ter sido realizada nas amostras liofilizadas. Existe uma alta correlação negativa entre o teor de umidade do abacate e lipídios, já relatada por Tango et al. (2004). Segundo estes autores, o elevado conteúdo de umidade na polpa fresca constitui um obstáculo para a obtenção do óleo. Abacates 'Hass' possuem teor de umidade inferior a outras variedades, além de conter teor de lipídios superior a $18 \%$, sendo assim indicados para extração de óleo.

O teor de lipídios na polpa foi superior aos encontrados na casca e na semente. Gondim et al. (2005) encontraram valores de11,04 na casca do abacate, resultado este expresso de forma integral (100 $\mathrm{g}$ de amostra in natura das cascas de fruta). Os autores concluem que a casca de abacate é uma boa fonte de lipídios, pois fornece $14 \%$ da Ingestão diária recomendada (IDR), considerando os requerimentos nutricionais de um adulto. Os autores compararam os resultados com a Tabela de Composição Química de Alimentos (NEPA, 2011) e observaram valor igual para a polpa. Porém, neste trabalho, a variedade pesquisada não foi mencionada.

Os teores de fibra foram superiores na polpa e na casca de abacate em relação à semente. Gondim et al. (2005) encontraram valores idênticos na casca e na polpa de abacate. Salgado et al. (2008) encontraram uma quantidade apreciável de fibra, $4,85 \%$, em abacate 'Margarida', e segundo estes autores as fibras podem contribuir para a prevenção de certas enfermidades, como diverticulite, câncer de cólon, obesidade, problemas cardiovasculares e diabetes. Além disso, o alto teor de fibras da polpa permite que o farelo resultante da extração do óleo possa ser utilizado na elaboração de produtos de panificação (biscoitos e pães) e massas alimentícias, ampliando a oferta de produtos.

Os valores de cinzas também foram superiores na polpa, e os valores de $\mathrm{pH}$ não diferiram entre as partes do fruto, porém a acidez foi superior na casca. $\mathrm{O}$ teor de umidade determinado antes e após a 
liofilização das amostras foi maior na polpa, seguido, respectivamente, da casca e semente.

\section{MINERAIS}

Dentre os macronutrientes analisados, o potássio é o que se encontra em maior quantidade no abacate 'Hass'(Tabela 2). A polpa e a casca de abacate não diferiram quanto ao teor de potássio e magnésio, apresentando valores superiores, sendo estes valores superiores ao da semente. Os teores de Ca na casca foram $65,52 \%$ maiores em relação aos encontrados na semente e na polpa do abacate.

Quanto aos micronutrientes, a polpa mostrou teores superiores de cobre em relação à casca, seguida da semente. Os teores de ferro foram 63,27\% maiores na casca em relação à polpa.O menor teor deste elemento mineral foi encontrado na semente. Já os teores de zinco da casca e da polpa foram superiores ao encontrado no caroço e não diferiram estatisticamente.

Cobre e zinco são denominados elementostraço e participam de diversas reações no organismo, diretamente ou como cofatores de enzimas, sendo considerados essenciais (ANDRADE et al., 2004). Os mesmos autores encontraram teor médio de cobre e zinco em amostras cruas de leguminosas, respectivamente, de $0,75 \mathrm{mg} \%$ e 3,2 $\mathrm{mg} \%$. Os autores concluem que, ao ser consumida uma porção média de leguminosas, cerca de $50 \mathrm{~g}$, a mesma fornece aproximadamente $19 \%$ e $10 \%$ das necessidades diárias de cobre e zinco, respectivamente, para um homem adulto, segundo a RDA (Recommended Dietary Allowance). Os teores destes elementos encontrados nas partes do abacate foram superiores aos das leguminosas relatadas na literatura, sendo, portanto, uma boa fonte destes minerais.

$\mathrm{O}$ enxofre foi o micronutriente que apresentou menores teores nas partes avaliadas. Já o boro foi o micronutriente que apresentou maiores teores em relação aos demais, sendo superior na polpa, seguido da casca e da semente de abacate.

Gondim et al. (2005) determinaram a composição centesimal de 7 elementos minerais com importância nutricional $(\mathrm{Ca}, \mathrm{Cu}, \mathrm{Fe}, \mathrm{K}, \mathrm{Mg}$, $\mathrm{Na}$ e $\mathrm{Zn}$ ) em 7 tipos diferentes de cascas de frutas: abacate, abacaxi, banana, mamão, maracujá, melão e tangerina, e neste caso os autores verificaram que a casca sempre apresentou valores superiores em todos os nutrientes analisados em relação à parte comestível.

\section{COMPOSTOS FENÓLICOS TOTAIS E ATIVIDADE ANTIOXIDANTE}

Os compostos fenólicos presentes nos vegetais são os principais responsáveis pela atividade antioxidante (KUSKOSKI et al., 2005; SANTOS et al., 2008). Constituem uma grande classe de fitoquímicos alimentares e encontram-se distribuídos entre as distintas partes das plantas; porém, sua maior concentração está nas frutas, hortaliças e em seus derivados (KARAKAYA, 2004). A Tabela 3 apresenta o teor de compostos fenólicos totais encontrados nas amostras.

A avaliação dos extratos pelo método de Folin-Ciocalteu mostrou que a casca e a semente possuem maior teor de compostos fenólicos quando comparados à polpa, apresentando 63,$5 ; 57,3$ e 3,3 $\mathrm{mg} \mathrm{GAE} / \mathrm{g}$, respectivamente. Torres et al. (1987), ao analisarem o teor de compostos fenólicos em quatro variedades de abacate, verificaram que os maiores teores destes compostos se encontravam na semente.

Segundo Gobbo-Neto e Lopes (2007), existem vários fatores que podem interferir no conteúdo de metabólitos secundários nas plantas, dos quais os compostos fenólicos fazem parte. Dentre estes, estão a sazonalidade, temperatura, disponibilidade hídrica, radiação ultravioleta, adição de nutrientes, poluição atmosférica, danos mecânicos e ataque de patógenos. Também se destacam as diferenças nas condições agronômicas e ambientais, que podem afetar o conteúdo de fenólicos presente nos vegetais, além da informação genética (variedade) que também afeta diretamente na quantidade de tais compostos (LLORACH et al., 2008).

Wang et al. (2010), ao analisarem o teor de compostos fenólicos em oito cultivares de abacate, verificaram que, em todas as cultivares, a casca e a semente apresentaram elevados teores destes compostos. Este alto teor de compostos fenólicos na semente de abacate também foi encontrado no trabalho de Soong e Barlow (2004).

O conteúdo de compostos fenólicos pode variar nas diferentes partes dos frutos, principalmente quando se comparam a casca e a polpa. Normalmente na casca, observa-se maior concentração destes compostos. Provavelmente, os compostos fenólicos tendem a se acumular na epiderme dos frutos, como forma de proteção à radiação ultravioleta e em defesa a determinados patógenos e predadores (DIXON; PAIVA, 1995).

Tango et al. (2004) apontam que a principal dificuldade na utilização dos caroços de abacate é a presença de substâncias fenólicas, que apresentam toxidez para animais monogástricos. Ichimaru et 
al.(1982) demonstraram que, pela extração dessas substâncias com etanol, os caroços poderiam ser usados na alimentação dos animais monogástricos.

Em relação à atividade antioxidante pelo método sequestro do radical livre DPPH das amostras de abacate, novamente a casca e a semente apresentaram maiores valores (Tabela 3). A atividade antioxidante em equivalente trolox na casca foi de 310,0 $\mu \mathrm{mol} \mathrm{TE} / \mathrm{g}$, e na semente, 410,7 $\mu \mathrm{mol} \mathrm{TE} / \mathrm{g}$, sendo que a polpa apresentou um valor igual a 8,1 $\mu \mathrm{mol} \mathrm{TE} / \mathrm{g}$. Estudos recentes têm demonstrado que as frutas são ricas em muitos nutrientes e compostos antioxidantes, sendo que há relatos de que esses constituintes se concentram majoritariamente nas cascas e nas sementes (MELO et al., 2008).

Wang et al. (2010), estudando oito cultivares de abacates, verificaram que amostras da casca e da semente das diferentes cultivares apresentaram maior atividade antioxidante. Para a mesma cultivar em estudo, encontraram valores menores para a polpa, casca e semente $(1,3 \mu \mathrm{mol} \mathrm{TE} / \mathrm{g} ; 189,8$ $\mu \mathrm{mol} \mathrm{TE} / \mathrm{g} ; 164,6 \mu \mathrm{mol} \mathrm{TE} / \mathrm{g}$, respectivamente). A composição do fruto pode variar quando são colhidos em diferentes épocas do ano (VILLA-RODRÍGUEZ, 2011) e também devido aos diferentes estágios de amadurecimento, localização geográfica, condições ambientais, entre outros.

A capacidade antioxidante da polpa, casca e semente, avaliada pelo método de captura do radical $\mathrm{ABTS}^{+}$(Tabela 3), mostrou que a casca e a semente apresentaram novamente as maiores atividades $(791,5 \mu \mathrm{mol} \mathrm{TEAC} / \mathrm{g}$ e $645,8 \mu \mathrm{mol}$ TEAC/g) quando comparadas à polpa $(15,2 \mu \mathrm{mol}$ $\mathrm{TEAC} / \mathrm{g}$ ).

Soong e Barlow (2004), ao analisarem a atividade antioxidante da polpa e da semente de abacate, jaca, longan, manga e tamarindo pela metodologia do $\mathrm{ABTS}^{+}$, verificaram que as sementes apresentaram maior atividade antioxidante quando comparadas à polpa. Para os frutos de abacate,encontraram atividades bem próximas para a semente e para a polpa $(725,0 \mu \mathrm{mol}$ TEAC/g e 13,1 $\mu \mathrm{mol} \mathrm{TEAC} / \mathrm{g})$, os quais poderiam ser aproveitados devido ao grande potencial antioxidante.

TABELA 1- Caracterização física e química nas diferentes partes do abacate 'Hass'.

\begin{tabular}{ccccc}
\hline Mineral & Polpa & Casca & Semente & Valor de p \\
\hline Umidade polpa fresca & $69,85 \pm 11,49 \mathrm{~A}$ & $61,43 \pm 9,16 \mathrm{~B}$ & $60,27 \pm 10,15 \mathrm{~B}$ & $<0,001$ \\
Umidade polpa & & & & \\
liofilizada & $13,99 \pm 0,25 \mathrm{~B}$ & $9,87 \pm 0,11 \mathrm{C}$ & $14,55 \mathrm{~A}$ & $<0,001$ \\
Lipídios* & $64,09 \pm 1,60 \mathrm{~A}$ & $2,18 \pm 0,17 \mathrm{~B}$ & $3,32 \pm 0,96 \mathrm{~B}$ & $<0,001$ \\
Fibras* & $3,81 \pm 0,16 \mathrm{~A}$ & $1,29 \pm 0,09 \mathrm{~B}$ & $3,97 \pm 0,08 \mathrm{~A}$ & $<0,001$ \\
Proteína* & $1,27 \pm 0,07 \mathrm{~A}$ & $0,17 \pm 0,02 \mathrm{~B}$ & $0,14 \pm 0,03 \mathrm{~B}$ & $<0,001$ \\
Açúcares redutores* & $0,26 \pm 0,08 \mathrm{C}$ & $0,65 \pm 0,06 \mathrm{~B}$ & $1,64 \pm 0,08 \mathrm{~A}$ & $<0,001$ \\
Minerais* & $11,56 \pm 0,56 \mathrm{~A}$ & $2,15 \pm 0,01 \mathrm{~B}$ & $2,81 \pm 0,13 \mathrm{~B}$ & $<0,001$ \\
\hline
\end{tabular}

Letras maiúsculas comparam médias para as variáveis na linha.

*Determinações nas amostras liofilizadas

TABELA 2- Teores de macronutrientes $\left(\mathrm{g} \mathrm{kg}^{-1}\right)$ e micronutrientes $\left(\mathrm{mg} \mathrm{kg}^{-1}\right)$ nas diferentes partes de abacate 'Hass'.

\begin{tabular}{ccccc}
\hline Mineral & Polpa & Casca & Semente & Valor de p \\
\hline $\mathbf{N}$ & $8,14 \pm 1,09 \mathrm{~A}$ & $8,45 \pm 0,16 \mathrm{~A}$ & $6,35 \pm 0,16 \mathrm{~B}$ & 0,014 \\
$\mathbf{P}$ & $2,10 \pm 0,04 \mathrm{~A}$ & $1,02 \pm 0,05 \mathrm{~B}$ & $0,94 \pm 0,06 \mathrm{~B}$ & $<0,001$ \\
$\mathbf{K}$ & $17,37 \pm 1,46 \mathrm{~A}$ & $16,60 \pm 1,93 \mathrm{~A}$ & $9,03 \pm 0,74 \mathrm{~B}$ & $<0,001$ \\
$\mathbf{C a}$ & $0,30 \pm 0,0 \mathrm{~B}$ & $0,87 \pm 0,12 \mathrm{~A}$ & $0,30 \pm 0,0 \mathrm{~B}$ & $<0,001$ \\
$\mathbf{M g}$ & $0,87 \pm 0,06 \mathrm{~A}$ & $0,87 \pm 0,12 \mathrm{~A}$ & $0,70 \pm 0,0 \mathrm{~B}$ & 0,04 \\
$\mathbf{S}$ & $3,1 \pm 0,07 \mathrm{~A}$ & $1,66 \pm 0,02 \mathrm{~B}$ & $1,55 \pm 0,04 \mathrm{~B}$ & $<0,001$ \\
$\mathbf{B}$ & $104,96 \pm 1,16 \mathrm{~A}$ & $84,91 \pm 3,65 \mathrm{~B}$ & $29,93 \pm 1,67 \mathrm{C}$ & $<0,001$ \\
$\mathbf{C u}$ & $12,67 \pm 0,58 \mathrm{~A}$ & $9,00 \pm 0,00 \mathrm{~B}$ & $7,00 \pm 0,00 \mathrm{C}$ & $<0,001$ \\
$\mathbf{F e}$ & $12,00 \pm 1,0 \mathrm{~B}$ & $32,67 \pm 2,08 \mathrm{~B}$ & $8,67 \pm 1,16 \mathrm{~B}$ & $<0,001$ \\
$\mathbf{M n}$ & $6,33 \pm 0,58 \mathrm{~B}$ & $12,67 \pm 1,16 \mathrm{~A}$ & $3,00 \pm 0,00 \mathrm{C}$ & $<0,001$ \\
$\mathbf{Z n}$ & $21,67 \pm 1,53 \mathrm{~A}$ & $29,00 \pm 6,08 \mathrm{~A}$ & $10,33 \pm 0,58 \mathrm{~B}$ & $<0,002$ \\
\hline
\end{tabular}

Letras maiúsculas comparam médias para as variáveis na linha. 
TABELA 3- Compostos fenólicos totais (eq. de ácido gálico (GAE), atividade antioxidante pelo sequestro do radical livre ABTS, expressos como valor TEAC (capacidade antioxidante total do composto equivalente ao Trolox), e atividade antioxidante equivalente ao Trolox, nos extratos etanólicos $(80 \%)$ da polpa, casca e semente de abacate 'Hass'.

\begin{tabular}{cccc}
\hline Amostras & $\begin{array}{c}\text { Compostos Fenólicos Totais } \\
\mathbf{m g} \text { GAE/g }\end{array}$ & $\begin{array}{c}\text { Atividade Antioxidante } \\
\text { DPPH }(\boldsymbol{\mu m o l ~ T E} / \mathbf{g})\end{array}$ & $\begin{array}{c}\text { Atividade Antioxidante } \\
\text { ABTS }(\boldsymbol{\mu m o l ~ T E A C} / \mathbf{g})\end{array}$ \\
Polpa & $3,3 \pm 0,7 \mathrm{~B}$ & $8,1 \pm 1,9 \mathrm{~B}$ & $15,2 \pm 1,2 \mathrm{~A}$ \\
Casca & $63,5 \pm 7,2 \mathrm{~A}$ & $310,0 \pm 36,9 \mathrm{~A}$ & $791,5 \pm 35,9 \mathrm{~A}$ \\
Semente & $57,3 \pm 2,7 \mathrm{~A}$ & $410,7 \pm 35,8 \mathrm{~A}$ & $645,8 \pm 17,9 \mathrm{~A}$ \\
\hline
\end{tabular}

* Valores das médias das triplicatas \pm desvio- padrão/Médias seguidas de letras diferentes na linha diferem estatisticamente $(\mathrm{p}<0,05)$, pelo teste de Tukey.

\section{CONCLUSÃO}

Acasca do abacate 'Hass' pode ser considerada como fonte alternativa de nutrientes, sugerindo seu aproveitamento na alimentação, principalmente pela sua composição em minerais. No entanto, ainda se fazem necessários estudos de fatores antinutricionais e avaliação de produtos na forma de farinhas. A maior atividade antioxidante na casca e na semente, em relação à polpa do abacate 'Hass', sugerem a utilização destes dois resíduos como fontes de antioxidantes naturais para aplicação na indústria de alimentos em substituição aos antioxidantes sintéticos.

\section{AGRADECIMENTO}

À Coordenação de Aperfeiçoamento de Pessoal de Nível Superior (CAPES) e à Fundação de Amparo à Pesquisa do Estado de São Paulo (FAPESP), Processo no 2010/52671-8.

\section{REFERÊNCIA}

ANDRADE, E.C.B.; BARROS, A.M.; MA GALH ÃES, A.C.P.; CASATROS, L.L.C.;TAKESEI, I.Comparação dos teores de cobre e zinco em leguminosas cruas e após serem processadas termicamente em meio salino e aquoso. Ciência e Tecnologia de Alimentos, Campinas, v.24, n.3, p.316-318, 2004.

DAIUTO, E. R.; VIEITES, R. L.; TREMOCOLDI, M. A.; VILEIGAS, D. F. Estabilidade físico-química de um produto de abacate (Persea American Mill.) conservado pelo frio. Alimentos e Nutrição, Araraquara, v. 21, p. 97-105, 2010.
DAIUTO, E.R.; SIMON, J.W.; VIEITES, R.L.; CARVALHO, L.R.; RUSSO, V.C. Aceitabilidade e viabilidade tecnológica da elaboração de dois produtos de abacate 'Hass'. Revista Iberoamericana de Tecnología Postcosecha, México, v. 13, n.1, p.66-75, 2012.

DIXON, R.A.; PAIVA, N.I. Stress-induced phenylpropanoid metabolism. The Plant Cell, Rockville, v.7, p.1085-1097, 1995.

GOBBO-NETO, L.; LOPES, N.P. Plantas medicinais: fatores de influência no conteúdo de metabólitos secundários. Química Nova, São Paulo, v.30, n.2, p.374-381, 2007

GÓMEZ-LÓPES, V.M. Fruit characterization of high oil content avocado varieties. Scientia Agricola, Piracicaba, v.59, n.2, p.403-406, 2002.

GONDIM, J.A.M.; MOURA, M.F.V.; DANTAS, A.S.; MEDEIROS, R.L.S.; SANTOS, K.M. Composição centesimal e de minerais em cascas de frutas. Ciência e Tecnologia de Alimentos, Campinas, v.25, n.4, p.825-827, 2005.

ICHIMARU, D. L.; SALES, A. M.; IADEROZA, M.; BALDINI, V. L. S.Estudo dos fatores antinutricionais do caroço de abacate (Persea americana Mill. cv. Wagner). Coletânea do ITAL, Campinas, v. 12, p. 67-83, 1982.

INSTITUTO ADOLFO LUTZ . Métodos físicoquímicos para análise de alimentos. São Paulo, 2008. p. 1020.

JAYAPRAKASHA, G. K.; PATIL, B. S. In vitro evaluation of the antioxidant activities in fruit extracts from citron and blood orange. Food Chemistry, London v. 101, n. 1, p. 410-418, 2007. 
KÄHKÖNEN, M.P.; HOPIA, A.I.; VUORELA, H. J.; RAUHA, J.P.; KALEVI, P.; KUJALA, T.S.; HEINONEN, M. Antioxidant Activity of Plant Extracts Containing Phenolic Compounds. Journal of Agricultural and Food Chemistry, Easton, v. 47, n. 10, p. 3954-3962, 1999.

KARAKAYA, S. Bioavailability of phenolic compounds. Critical Reviews in Food Science and Nutrition, Boca Raton, v. 44, n. 6, p. 453-464, 2004.

KUSKOSKI, E. M.; ASUERO, G. A.; TRONCOSO, A. M.; MANCINI-FILHO, J.; FETT, R. Aplicação de diversos métodos químicos para determinar actividade antioxidante em polpa de frutos. Revista de Ciência e Tecnologia de Alimentos, Campinas, v. 25, n. 4, p. 726-732, 2005.

LEE, J.; KOO, N.; MIN, D. Reactive oxygen species, aging, and antioxidative nutraceuticals. Comprehensive Reviews in Food Science and Food Safety, Chicago, v.3, n.1, p. 21-33, 2004.

LLORACH, R.; MARTÍNEZ-SÁNCHEZ, A.; TOMÁS-BARBERÁN, F.A.; GIL, M.I.; FERRERES, F. Characterisation of polyphenols and antioxidant properties of five lettuce varieties and escarole. Food Chemistry, London, v.108, p.1028-1038, 2008.

MALAVOLTA, E.; VITTI, G. C.; OLIVEIRA, S.A. Avaliação do estado nutricional de plantas: princípios e aplicações. 2. ed. Piracicaba: Potafós, 1997. 319p.

MELO, E. A.; MACIEL, M. I. S.; LIMA, V. A. G. L.; NASCIMENTO, R. J. Capacidade antioxidante de frutas. Revista Brasileira de Ciências Farmacêuticas, São Paulo,v. 44, n. 2, p.193-201, 2008.

MELO, P. S. Composição química e atividade biológica de resíduos agroindustriais. 2010. 100f. Dissertação (Mestrado em Ciência e Tecnologia de Alimentos) - Escola Superior de Agricultura Luiz de Queiroz, Universidade de São Paulo, Piracicaba, 2010 .

MENSOR, L. L.; MENEZES, F. S.; LEITÃO, G.G.; REIS, A.S.; DOS SANTOS, T.C.; COUBE, C.S.; LEITÃO, S.G. Screening of Brazilian plant extracts for antioxidant activity by the use of DPPH free radical method. Phytotherapy Research, London, v. 15, n. 2, p. 127-130, 2001.
NELSON, N. A. Pthotometric adaptation of the Somogy method for the determination of glucose. Journal of Biological Chemistry, Baltimore, n. 153, p. 375-380, 1944.

NEPA- Núcleo de Etudos e Pesquisa em Alimentação. Tabela brasileira de composição de alimentos. TACO. 4.ed. Campinas: UNICAMP, 2011. 161p.

PINTO, N.A.V.D.; CARVALHO, V. D. de; BOTElHO, V. A. V. A.; MORAES, A. R. de. Determinación Del potencial de fibras dietéticas em lãs hojas de taioba (Xanthosoma sagittifolium Schott). Revista Alimentaria, Madrid, v.5, n.312, p.87-90, 2000.

RE, R.; PELLEGRINI, N.; PROTEGGENTE, A.; PANNALA, A.; YANG, M.; RICE-EVANS, C. Antioxidant activity applying an improved ABTS radical cation decolorization assay. Free Radical Biology \& Medicine, New York, v. 26, n. 9-10, p. 1231- 1237, 1999.

SALGADO, J.M.;DANIELI, F.; REGINATOD'ARCE, M.A.B.; FRIAS, A.; MANSI, D.N. O óleo de abacate (Persea americana Mill) como matéria-prima para a indústria alimentícia. Ciência e Tecnologia de Alimentos, Campinas, v.28, p.20-26, 2008. Suplemento

SANTOS, G. M.; MAIA, G. A.; SOUSA, P. H. M.; COSTA, J. M.C.;FIGUEIREDO, R. W.; PRADO, G. M. Correlação entre atividade antioxidante e compostos bioativos de polpas comerciais de açaí (Euterpe oleracea Mart). Archivos Latinoamericanos de Nutricion, Caracas, v. 58, n. 2, p. 187-192, 2008.

SAS. Statistical analysis system user's guide. Version 9. $13^{\text {th }}$ ed. Cary: SAS Institute, 2002.

SINGLETON, V. L.; ORTHOFER, R.; LAMUELA, R. M. Analysis of total phenols and other oxidation substrates and antioxidants by means of FolinCiocalteau reagent, Methods of Enzymology, New York, v. 299, p. 152-178, 1999.

SOMOGY, M. Determination of blood sugar. Journal of Biological Chemistry, Baltimore, $n$. 160, p. 69-73, 1945.

SOONG, Y.-Y.; BARLOW, P. J. Antioxidant activity and phenolic content of selected fruit seeds. Food Chemistry, Barking, v. 88, n. 3, p. 411-417, 2004. 
TANGO, J. S.; CARVALHO, C. R. L.; SOARES, N. B. Caracterização física e química de frutos de abacate visando a seu potencial para extração de óleo. Revista Brasileira de Fruticultura, Jaboticabal, v.6, n.1, p,17-23, 2004.

TORRES, A.M.; MAU-LASTIVICKA, T.; REZAAIYAN, R. Total Phenolics and HighPerformance Liquid Chromatography of Phenolic Acids of Avocado. Journal of Agricultural and Food Chemistry, Easton, v. 35, n. 6, 1987.

VILLA-RODRÍGUEZ, J.;MOLINA-CORRAL, F.J.; AYLA-ZAVALA, J.F.; OLIVAS, G.I.; GONZÁLEZAGUILAR, G. A. Effect of maturity stage on the content of fatty acids and antioxidant activity of 'Hass' avocado. Food Research International, Barking, v.44, 1.231-1.237, 2011.
WANG, W.; TERRELL, R.; BOSTIC, L.G. Antioxidant capacities, procyanidins and pigments in avocados of different strains and cultivars. Food Chemistry, Barking, v.122, p. 1.193-1.198, 2010.

WENG, X. C.; WANG, W. Antioxidant activity of compounds isolated from Salvia plebeia Food Chemistry, London, v. 71,n.4, p. 489-493, 2000. 